\title{
Design of SVC Controller Based on Improved Biogeography-Based Optimization Algorithm
}

\author{
Feifei Dong, Dichen Liu, Jun Wu, Bingcheng Cen, Haolei Wang, Chunli Song, and Lina Ke
}

School of Electric Engineering, Wuhan University, Wuhan 430072, China

Correspondence should be addressed to Jun Wu; flysky007dong@163.com

Received 18 January 2014; Revised 5 April 2014; Accepted 12 April 2014; Published 8 May 2014

Academic Editor: Hongjie Jia

Copyright (C) 2014 Feifei Dong et al. This is an open access article distributed under the Creative Commons Attribution License, which permits unrestricted use, distribution, and reproduction in any medium, provided the original work is properly cited.

\begin{abstract}
Considering that common subsynchronous resonance controllers cannot adapt to the characteristics of the time-varying and nonlinear behavior of a power system, the cosine migration model, the improved migration operator, and the mutative scale of chaos and Cauchy mutation strategy are introduced into an improved biogeography-based optimization (IBBO) algorithm in order to design an optimal subsynchronous damping controller based on the mechanism of suppressing SSR by static var compensator (SVC). The effectiveness of the improved controller is verified by eigenvalue analysis and electromagnetic simulations. The simulation results of Jinjie plant indicate that the subsynchronous damping controller optimized by the IBBO algorithm can remarkably improve the damping of torsional modes and thus effectively depress SSR, and ensure the safety and stability of units and power grid operation. Moreover, the IBBO algorithm has the merits of a faster searching speed and higher searching accuracy in seeking the optimal control parameters over traditional algorithms, such as BBO algorithm, PSO algorithm, and GA algorithm.
\end{abstract}

\section{Introduction}

Series capacitor compensation has been widely implemented in order to improve the transmission capacity of transmission line, but the employment of series compensators could potentially lead to subsynchronous resonance (SSR). SSR is an abnormal electric power system operation condition where the electrical network exchanges energy with a turbine generator (T-G) at one or more frequencies below the synchronous frequency of the system [1]. Subsynchronous resonance creates instability within a system's oscillation, which may directly result in serious damages to the rotor shafting of large steam turbine generators directly. Since 1970, there have been many accidents caused by SSR and this has seriously jeopardized the safe operation of power systems [2].

As the first batch of FACTS devices was put into practical use, in 1980, static var compensator (SVC) was recommended as one of the most effective devices for suppressing SSO by Subsynchronous Oscillation Working Group (SSRWG) founded by IEEE $[3,4]$. The subsynchronous damping controller (SSDC) designed for SSR can effectively suppress subsynchronous resonance; however, the common design methods of the controller, such as mode of control [5] and
PID control [6], are not able to be well adapted to the characteristics of a time-varying and nonlinear power system, which causes the designed controller to be incapable of obtaining a good inhibition effect.

The cosine migration model, the improved migration operator, and the mutative scale of chaos and Cauchy mutation strategy are introduced into the improved biogeographybased optimization algorithm based on the basic BBO algorithm in this paper. The series compensation output system of Jinjie plant is regarded as a research objective to design an optimal SVC subsynchronous damping controller with the proposed improved algorithm, which improves the modal damping of unit torsional vibration significantly, and suppresses subsynchronous resonance effectively.

\section{Mechanism of SVC Suppressing Subsynchronous Resonance}

The static var compensator (SVC) can be seen as a reactive component with variable admittance value. Its main component is the thyristor controlled reactor (TCR) and the reactive 


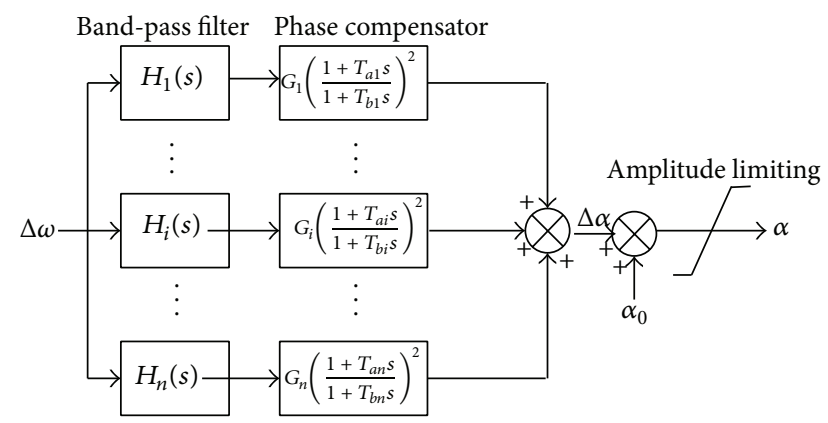

FIGURE 1: Structure diagram of SSDC.

power absorption of the SVC can be adjusted by the trigger angle of the thyristor.

By applying SVC to suppress subsynchronous resonance, if the reactive current of SVC is modulated to obtain an angle deviation of $180^{\circ}$ with respect to the generator rotor speed, the positive damping would be guaranteed [7]. As the rotor speed increases, the decrease of the inductive current in SVC may lead to the increase of the generator's output power. For a constant mechanical input, the increase would cause the decease of the rotor's kinetic energy, which would eventually result in the reduction of rotor speed [8].

The structure diagram of SVC-SSDC is shown in Figure 1 [9]. Here, $\Delta \omega$ is per unit of the generator rotor's angular velocity deviation. $\Delta \omega$ is screened as the $n$ oscillatory components, respectively, corresponding to the shaft modes through a band-pass filter, which are then made to be the increment of trigger angle, $\Delta \alpha$. Moreover, it is combined with the steadystate instruction of the triggering angle $\alpha_{0}$, which is turned to be the instruction of the triggering angle changing with the subsynchronous resonance signal after the unit of amplitude limiting. This causes the SVC to increase or decrease the reactive power as needed, so that the electrical damping of system is improved and subsynchronous resonance is suppressed.

There are three parameters, namely, $G_{i}, T_{a i}$, and $T_{b i}$. In each modal $i, 3 n$ parameters should be designed according to $n$ shafting oscillation mode, which has a decisive influence on the performance of SVC. The IBBO algorithm is adopted to design and set these parameters.

\section{BBO Algorithm and Its Improvement}

3.1. The Principle of Basic BBO Algorithm. $\mathrm{BBO}$ algorithm is a new type of evolutionary algorithm that applies the biogeography theory to solve optimization problems. Its basic idea is as follows: a number of relatively independent habitats are constructed as candidate solutions. The information sharing is realized based on the species' migration between habitats and the information update is realized by the species' mutation. In this way, the fitness of the habitat is improved and the optimal solution of the problem is obtained [10, 11].

In $\mathrm{BBO}$ algorithm, the habitat suitability index (HIS) is used to describe the suitable extent of the habitat for creatures

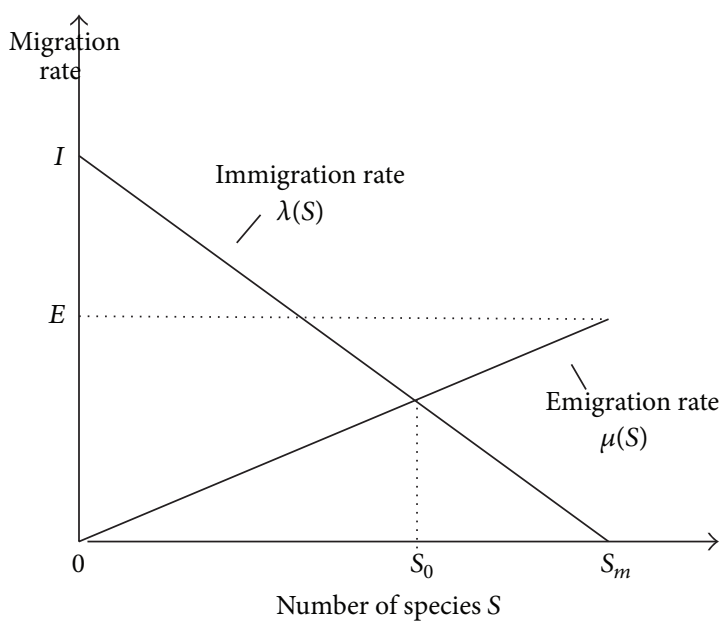

FIgURE 2: Species migration model.

and the suitable index vectors (SIV) of habitat are formed by the factors associated with HIS. The algorithm primarily includes migration and mutation operations [12-15].

3.1.1. Migration Operation. The migration operation is used for information exchange with other habitats and the searching of the solution space in a wide area. The common species' migration model of single habitat is shown in Figure 2.

In Figure 2, I denotes the largest immigration rate, $E$ is the biggest emigration rate, and $S_{0}$ and $S_{m}$ express balance and saturation point, respectively. The calculation method of $\lambda(S)$ and $\mu(S)$ is shown in the following:

$$
\begin{aligned}
& \lambda(S)=I \times\left(1-\frac{S}{S_{m}}\right), \\
& \mu(S)=E \times \frac{S}{S_{m}} .
\end{aligned}
$$

3.1.2. Mutation Operation. Mutation operation is used to increase the diversity of population. The probability of the habitat with number of species $S$ is written as $P_{s}$. The variation rate is obtained with the following formula:

$$
M(S)=M_{\max }\left(1-\frac{P_{s}}{P_{m}}\right),
$$

where $M_{\max }$ is the biggest mutation rate, a parameter set in accordance with the requirements of different users, and $P_{m}$ is the maximum of $P_{s}$.

$\lambda(S)$ and $\mu(S)$ are simplified as $\lambda_{s}$ and $\mu_{s}$, respectively, and $P_{s}$ is defined as follows:

$$
P_{s}= \begin{cases}-\left(\lambda_{s}+\mu_{s}\right) \times P_{s}+\mu_{s+1} P_{s+1} & S=0 \\ -\left(\lambda_{s}+\mu_{s}\right) \times P_{s}+\lambda_{s-1} P_{s-1}+\mu_{s+1} P_{s+1} & 1 \leq S \leq S_{m}-1, \\ -\left(\lambda_{s}+\mu_{s}\right) \times P_{s}+\lambda_{s-1} P_{s-1} & S=S_{m} .\end{cases}
$$

Mutation operation causes the solution of lower HIS to improve by variation and causes the higher HIS to obtain the opportunity of improving their solutions [16]. 


\subsection{The Improvement of $B B O$ Algorithm}

3.2.1. The Improvement of Migration Model. Because of that the linear species migration model shown in Figure 2 cannot accurately simulate the material migration's complex process of the actual biological geographical environment and therefore the cosine migration model, which is more conforming to the nature, is used to calculate migration rate. The expression of immigration rate $\lambda(S)$ and emigration rate $\mu(S)$ is as follows:

$$
\begin{gathered}
\lambda(S)=\frac{I}{2}\left(\cos \left(\frac{S \pi}{S_{m}}\right)+1\right), \\
\mu(S)=\frac{E}{2}\left(-\cos \left(\frac{S \pi}{S_{m}}\right)+1\right) .
\end{gathered}
$$

In this migration model, when there are less or more species in the habitat, the immigration and emigration rates change smoothly, while when habitat has a certain number of species, they change relatively quickly.

3.2.2. The Improved Migration Operator. In $\mathrm{BBO}$ algorithm, the migration operation plays a key role in promoting the evolution of the algorithm. Since the traditional discrete migration operator is difficult to converge, the mixed migration operator is proposed in order to carry out the migration operation. The suitable index vectors (SIV) of $X_{j}$ in approaching habitat and the SIV of $X_{i}$ itself are combined with the weight factor, which would replace the original SIV. The specific expression is as follows:

$$
\Omega^{*}(\lambda, \mu): X_{i}(\mathrm{SIV})=\alpha \times X_{i}(\mathrm{SIV})+(1-\alpha) \times X_{j}(\mathrm{SIV}) .
$$

Here, $\Omega^{*}(\lambda, \mu)$ is the mixed migration operator and $\alpha$ is the weighting coefficient, defined as a constant or variable coefficient according to the actual situation.

The mixed migration operator not only ensures that the habitat with higher HIS is unabated in the migration operation, but it also causes the habitat with lower HIS to enjoy the habitat's SIV with higher HIS more, which is conducive to optimize the solution set and enhances the convergence of the algorithm.

\subsubsection{The Mutative Scale of Chaos and Cauchy Mutation} Strategy Is Introduced. The mutation strategy of $\mathrm{BBO}$ algorithm directly affects whether the algorithm will fall into local optimum and convergence precision. The mutative scale of chaos and Cauchy mutation strategy are introduced in this paper. In the early evolution of $\mathrm{BBO}$ algorithm, the ergodicity of chaos is used for random search in the vast space, which is helpful for solving the problem of local optimum and aids in convergence towards a global optimal solution. In the last stage of evolution, the center distribution characteristics of the Cauchy iteration are used for a careful search closely around the optimal solution, thus improving the convergence precision.
The segmented logistic chaotic iterative equation is adopted in this paper $[17,18]$ :

$$
z_{j}^{k+1}= \begin{cases}4 \times \mu \times z_{j}^{k} \times\left(0.5-z_{j}^{k}\right), & 0 \leq z_{j}^{k} \leq 0.5, \\ 4 \times \mu \times\left(z_{j}^{k}-0.5\right) \times\left(1-z_{j}^{k}\right), & 0.5 \leq z_{j}^{k} \leq 1,\end{cases}
$$

where $z_{j}$ is the $j$ th chaotic variable and $K$ is the iterations of the chaos iteration. When this meets $3.5699456 \leq \mu \leq 4$, the system enters into a chaotic state. Usually take $\mu=4$.

The distribution function of Cauchy distribution is as follows:

$$
P(x)=\frac{1}{2}+\frac{1}{\pi * \arctan x} .
$$

The formula of the mutative scale of chaos and Cauchy mutation is as follows:

$$
X_{j}^{\prime(k)}=\left(1-\lambda_{k}\right) X_{j}^{(k)}+\lambda_{k} P_{c}
$$

where $X_{j}^{\prime(k)}$ is the newly generated optimized individual and $X_{j}^{(k)}$ is the best individual obtained from the current optimization sequence. If the suitability of $X_{j}^{\prime(k)}$ is better than that of the original best individual, it will remain. Otherwise, it will be abandoned. $P_{c}$ is the chaos or Cauchy iteration variable mapped to the parameters of the search space. $\lambda_{k}$ is the variable scale factor, which can be obtained with the following formula:

$$
\lambda_{k}=1-\left|\frac{(k-1)}{k}\right|^{r}
$$

where $r$ is used to control the convergence speed.

The high probability area of chaos iteration distribution is close to the edge, while the high probability area of Cauchy distribution is near the center.

At the beginning of the $\mathrm{BBO}$ algorithm's evolution, the value of $k$ is smaller and $\lambda_{k}$ tends to be 1 and $P_{c}$ is selected as chaos iteration variable, which can make the algorithm jump out of a local convergence as soon as possible. At the end of the algorithm, the value of $k$ is bigger and $\lambda_{k}$ tends to be 0 and $P_{c}$ takes Cauchy iteration variable, which can fix the optimal solution and improve the accuracy of the algorithm.

\section{The Optimal Design of SVC-SSDC Based on IBBO Algorithm}

4.1. The Design Thought of SVC-SSDC Optimal Control. The optimal design of SVC-SSDC aims to suppress multimodal SSO adaptively and ensures that the parameters of the controller are reasonable and easy to implement. Therefore, 
the design problem of SSDC is converted into a nonlinear constrained optimization problem:

$$
\begin{array}{ll}
\max & f=\frac{1}{n} \times\|\mathbf{R}\|_{1} \\
\text { s.t. } & \mathbf{R}=\left(\eta_{1}, \eta_{2}, \ldots, \eta_{i}, \ldots, \eta_{n}\right)^{T} \\
& \|\mathbf{R}\|_{1}=\sum_{i=1}^{n} \eta_{i} \\
& \eta_{i}>0 \\
& \left|G_{k}\right| \leq G_{m k} \\
0 \leq T_{k} \leq T_{m k}
\end{array}
$$

where $\eta_{i}$ is the worst damping value of the modal $i, f$ is the performance function which is used to evaluate the control effect of SVC-SSDC, and $\|\mathbf{R}\|_{1}$ is 1 norm of vector $\mathbf{R}$. The constraint condition of $\eta_{i}>0$ is used to ensure that the optimally designed SVC-SSDC can suppress all modal of SSO, $G_{m k}=10.15$ is the maximum of gain $G_{k}$ 's absolute value, and $T_{m k}=0.1 \mathrm{~s}$ is the upper limit of time constant $T_{k}$.

4.2. The Optimal Design of SSDC's Parameters Based on IBBO Algorithm. The cosine migration model, the improved migration operator, and the mutative scale of chaos and Cauchy mutation strategy are introduced into IBBO algorithm to solve the multimodal, multimodel, and nonlinear constrained optimization problems shown in formula (11). The process algorithm is shown in Figure 3. The specific steps are as follows.

Step 1. The control parameters of BBO algorithm are initialized as follows: the size of population is set as $N=50, k_{\max }=$ 50 is the maximum number of iterations, the biggest mutation rate is set as $M_{\max }=0.01$, the maximum of immigration rate and emigration rate is set as $I=E=1$, the elite reserve $Z=2$, global mobility $P_{\bmod }=1$, and the coefficient for controlling the convergence rate is $r=5$. The modal parameters such as $G_{i}, T_{a i}$, and $T_{b i}$ are regarded as SIV of each habitat and the initial population $W$ which meets the constraint conditions is randomly generated in the searching scope of $G_{i}, T_{a i}$, and $T_{b i}$.

Step 2. The suitability index of the habitat is calculated and sorted. The individual optimal solution, $f_{\text {best }}$, is saved and then determine whether it meets the end condition. If it meets the end condition, the results are output and then converted to backbone grid scheme. In that case, the program is over. Otherwise, continue to Step 3.

Step 3. The cosine migration model is established. The habitat's species number, immigration rate, and emigration rate are calculated.

Step 4. The migration operation as formula (6) is performed in order to form a new population $W_{1}$. The suitability index of the habitat is recalculated and then the optimal solution $f_{\text {best }}$ is updated.

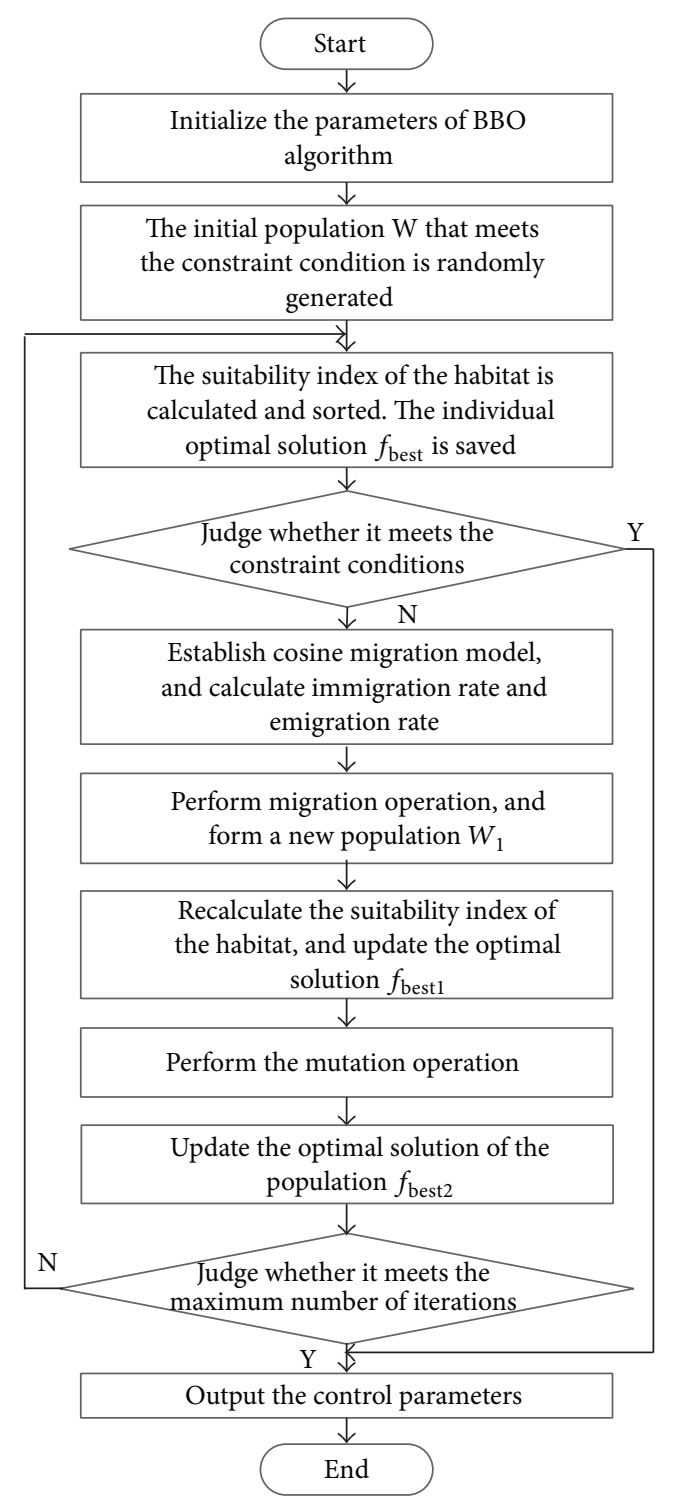

FIGURE 3: Flow chart of SSDC parameter optimization based on IBBO algorithm.

Step 5. The mutative scale of chaos and Cauchy mutation is carried out according to formula (9) and then the optimal solution of the population $f_{\text {best } 2}$ is updated.

Step 6. Determine whether it meets the maximum number of iterations. If this is satisfied, the results are output and then converted to core backbone grid scheme. In that case, the program is over. Otherwise, go to Step 2.

\section{The Simulation of Suppressing Jinjie Plant SSO by SVC}

5.1. Introduction of Jinjie Plant. The series compensated system of Jinjie plant is shown in Figure 4; its generator shafting models adopt four periods of the concentrated mass spring model. A detailed description is introduced in [19]. 


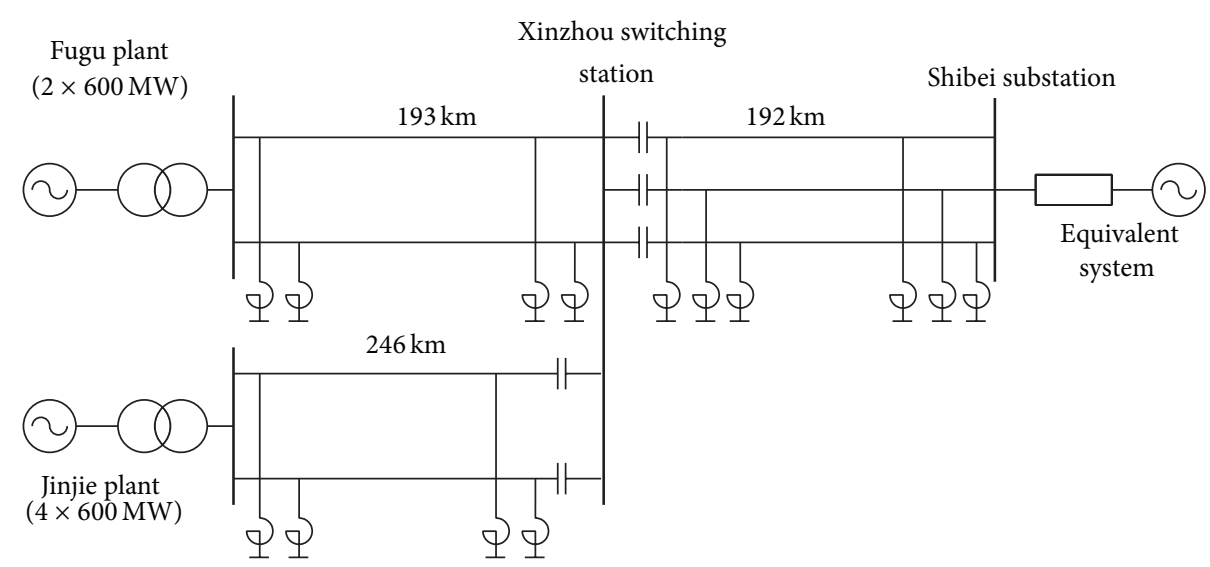

FIGURE 4: Single-line diagram of Jinjie power plant.

There are three modal frequencies in the shafting, namely, $13.19 \mathrm{~Hz}, 22.82 \mathrm{~Hz}$, and $28.19 \mathrm{~Hz}$. Modal 1 is stable and modal 2 is stable under normal operation, but it would present the phenomenon of instability or of being weakly damped under certain ways with a smaller output of generating units and a portion of the lines shut down. The damping of modal 3 is weakest, which is under the risk of subsynchronous resonance divergence in most operation regions.

5.2. The Simulation Analysis of Suppressing SSO. In order to suppress subsynchronous resonance, four parallel SVC are installed on the high side of the Jinjie plant's step-up transformer's bus bar and each SVC has a capacity of 100 MVar. Therefore, there are a total of 400 MVar. Since there are 3 oscillation modes in the generator shaft, 9 parameters should be set. IBBO algorithm is used to search for the optimal solution of the nine parameters after independently running 50 times. The suitability index of the optimal program is shown in Figure 5. In order to verify IBBO algorithm's performance, the traditional $\mathrm{BBO}$ algorithm, $\mathrm{PSO}$ algorithm, BACA algorithm, and GA algorithm are used to search the optimal scheme of the performance function $f$. Their population size and their maximum number of iterations are set as the same as IBBO algorithm. The optimal solution contrast of suitability index curves of each of the 4 algorithms is shown in Figure 5.

Figure 5 shows that IBBO algorithm can find better solutions with a lower number of iterations compared to the other three traditional algorithms. This has obvious advantages in convergence speed and convergence precision. The gain and time constant of SSDC's optimal solution solved by IBBO algorithm are, respectively, as follows: $G_{1}=10.15$, $G_{2}=10.15, G_{3}=10.15, T_{a 1}=0.0083, T_{a 2}=0.0827$, $T_{a 3}=0.0021, T_{b 1}=0.0182, T_{b 2}=0.0006$, and $T_{b 3}=0.0156$.

The real part of eigenvalues associated with the shaft modal under four operating conditions is shown in Table 1.

Operating condition 1 is as follows: six generators of Jinjie plant and Fugu plant are operating with rated power and lines and series compensation are normal.

Operating condition 2 is as follows: one generator of Jinjie plant is empty running; the other three generators of Jinjie

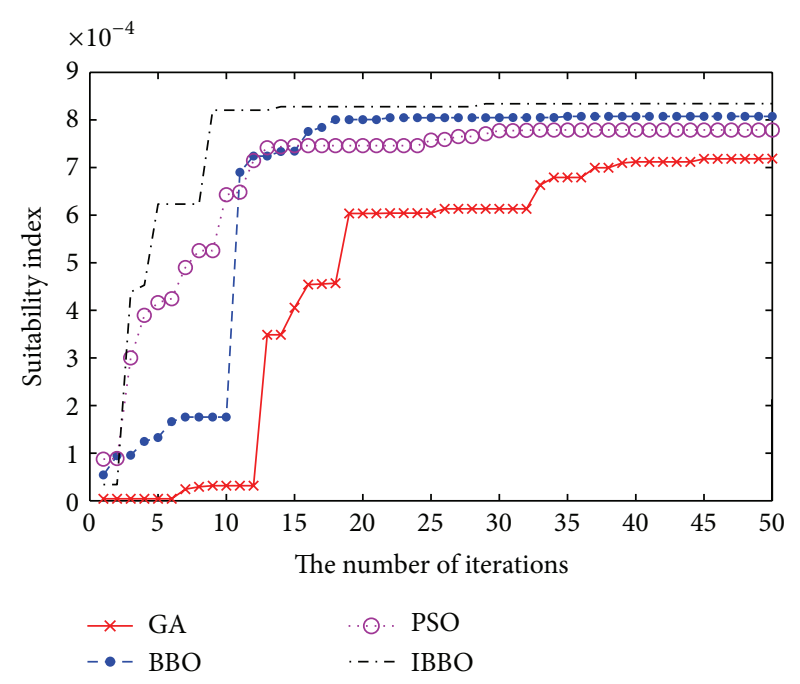

FIGURE 5: Contrast of suitability index curve of 4 kinds of algorithm optimal solution.

plant and the two units of Fugu plant are operating with 50\% rated power and lines and series compensation are normal.

Operating condition 3 is as follows: six generators of Jinjie plant and Fugu plant are operating with rated power, the two round lines of Xinzhou-Shibei are operating, and the rest of the lines and series compensation are normal.

Operating condition 4 is as follows: one generator of Jinjie plant is idle running, the other three generators of Jinjie plant and the two units of Fugu plant are operating with 50\% rated power, the two round lines of Xinzhou-Shibei are operating, and the rest lines and series compensation are normal.

Table 1 shows that modal 3 contains negative damping under the four kinds of operating conditions when there is no SVC, which would cause subsynchronous resonance. But all modals contain damping when SVC controlled by SSDC is installed. This indicates that the phenomena of subsynchronous resonance are suppressed.

In order to further verify the effect of optimal designed SVC damping controller, the simulation analysis is performed 
TABLE 1: Real parts of SSO shaft modes.

\begin{tabular}{|c|c|c|c|c|c|c|}
\hline \multirow{2}{*}{ Operating conditions } & \multicolumn{2}{|c|}{ Modal 1/13.19 Hz } & \multicolumn{2}{|c|}{ Modal $2 / 22.82 \mathrm{~Hz}$} & \multicolumn{2}{|c|}{ Modal 3/28.19 Hz } \\
\hline & Without SVC & With SVC & Without SVC & With SVC & Without SVC & With SVC \\
\hline 1 & -0.058 & -0.079 & -0.012 & -0.039 & 0.048 & -0.021 \\
\hline 2 & -0.070 & -0.085 & -0.016 & -0.045 & 0.053 & -0.020 \\
\hline 3 & -0.050 & -0.076 & -0.009 & -0.036 & 0.058 & -0.025 \\
\hline 4 & -0.068 & -0.083 & -0.006 & -0.041 & 0.057 & -0.023 \\
\hline
\end{tabular}

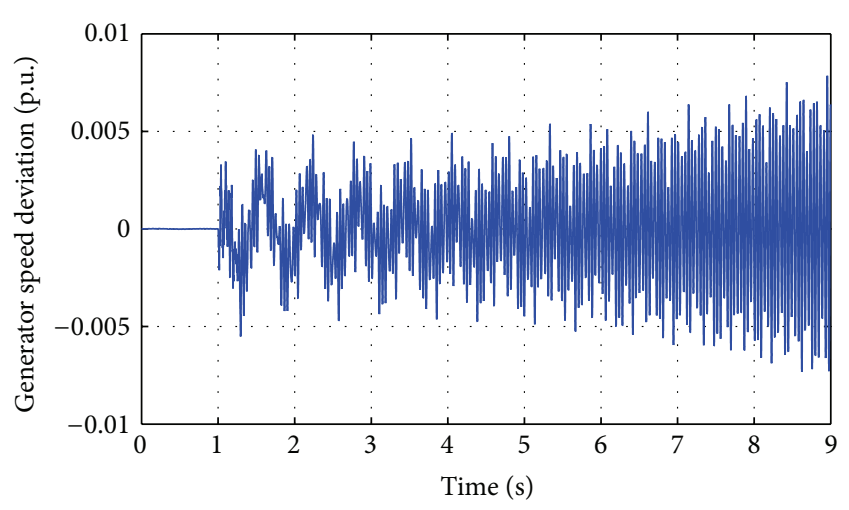

Figure 6: The generator speed deviation without SVC.

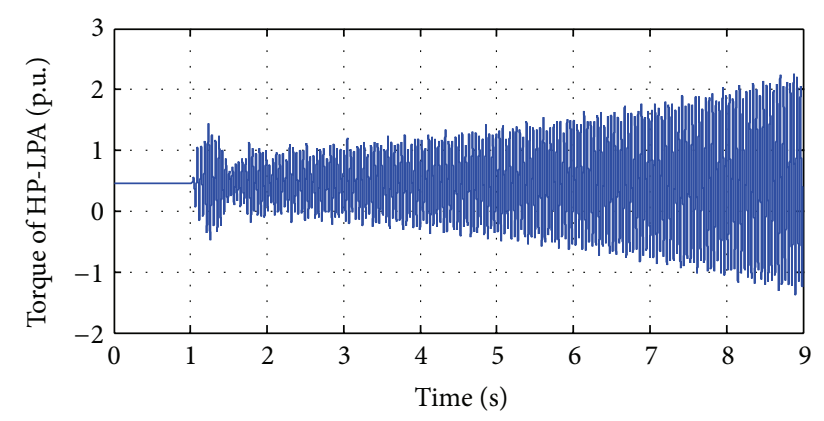

FIGURE 7: The torque of the generator shaft without SVC.

in PSCAD/EMTDC. Operating condition 2 is selected, threephase short-circuit fault is set at one round line of XinzhouShibei, and the fault lasts for $0.075 \mathrm{~s}$. The generator speed deviation and the torque of the generator shaft (take HP-LPA for an example) without SVC are shown in Figures 6 and 7, respectively.

As it can be seen from Figures 6 and 7, the generator torque between the shafts is divergent after a three-phase short-circuit fault, which endangers the safety of the generator shaft. The generator speed deviation and the generator torque between the shafts after the SVC damping controller optimally designed by IBBO algorithm are, respectively, shown in Figures 8 and 9 with the same operating condition and the same fault. In contrast, the generator speed deviation and the generator torque between the shafts after the SVC optimally designed by the PSO algorithm are shown in Figures 10 and 11, respectively.

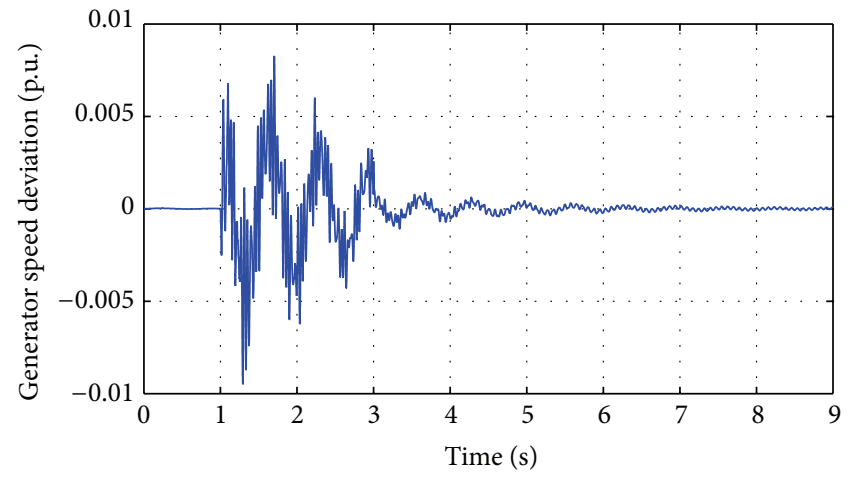

FIGURE 8: The generator speed deviation with SVC optimized by IBBO algorithm.

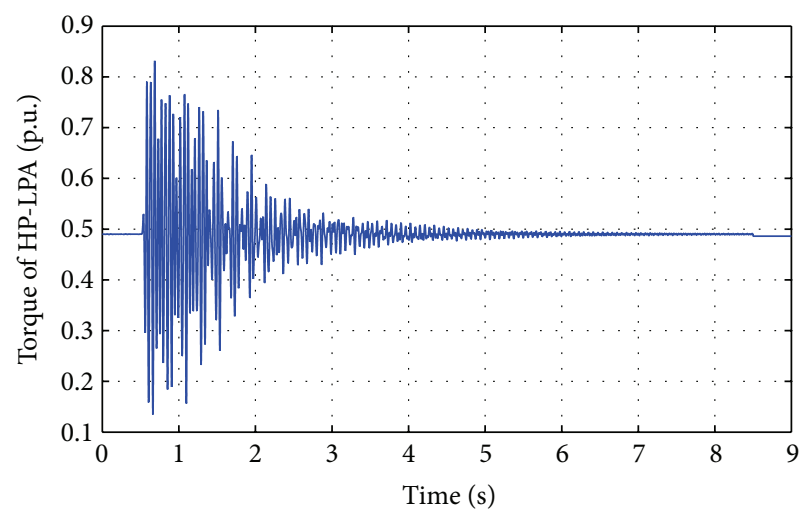

FIGURE 9: The inhibitory effect of SVC optimized by IBBO algorithm.

Figure 8 through Figure 11 show that the shaft torque presents the trend of stable attenuation after the SVC damping controller is installed. This indicates that subsynchronous resonance has been suppressed effectively. Compared with the inhibition effect of SVC damping controller optimized by PSO algorithm, the SVC damping controller optimized by IBBO algorithm causes the torque to dampen down in a shorter period of time. And the maximal amplitude of the torque is smaller, which can quickly quell oscillation when subsynchronous resonance occurs, thus reducing damage to the generator shaft.

The triggering angle curve of SVC designed optimally by IBBO algorithm is shown in Figure 12. It can be seen from Figure 12 that the triggering angle is in the limiting state at the beginning of the short-circuit because of the large torque 


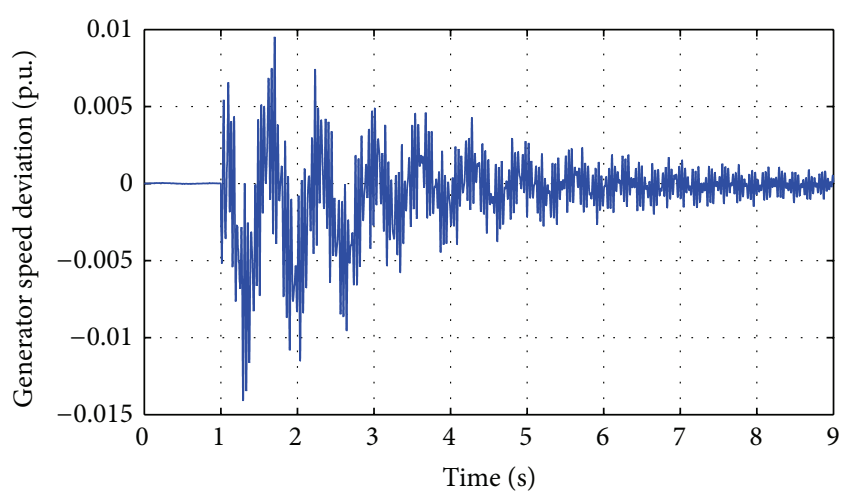

FIGURE 10: The generator speed deviation with SVC optimized by PSO algorithm.

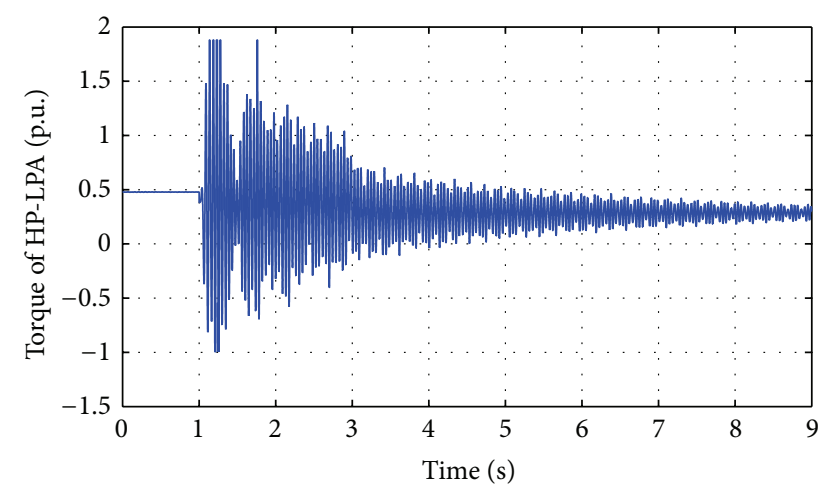

FIGURE 11: The inhibitory effect of SVC optimized by PSO algorithm.

and the triggering angle is turned to be stabilized after the subsidence of torque. This shows that SVC with this control modal can change its reactive power by tracking the change of the torque in order to achieve stable torque.

\section{Conclusion}

(1) The optimal designed SVC subsynchronous damping controller by IBBO algorithm can effectively restrain shafting torsional vibration with characteristics of a short reaction time and thorough robustness.

(2) IBBO algorithm converges quickly and it is not easy to fall into local optimum. It can search the optimal solution of control parameters with a fewer number of iterations. Moreover, it has the advantages of better convergence speed and convergence precision over traditional methods such as BBO algorithm, PSO algorithm, and GA algorithm.

(3) The proposed method is applied to the series compensated system of Jinjie plant. The effectiveness of the SVC subsynchronous damping controller optimized by IBBO algorithm is verified by the eigenvalue analysis and time domain simulation results.

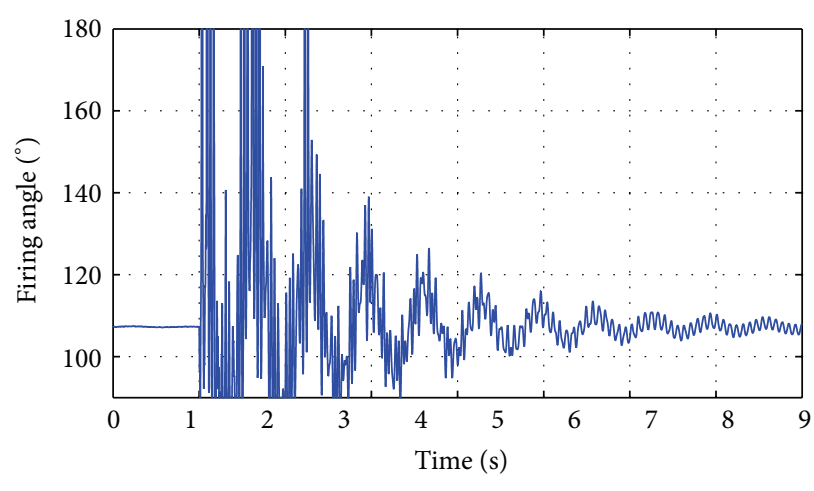

Figure 12: Curve of SVC's firing angle.

\section{Conflict of Interests}

The authors declare that there is no conflict of interests regarding the publication of this paper.

\section{Acknowledgment}

This work is funded by the Natural Science Foundation of China (51207114).

\section{References}

[1] IEEE Committee Report, "Reader's guide to subsynchronous resonance," IEEE Transactions on Power Systems, vol. 7, no. 1, pp. 150-157, 1992.

[2] C. A. R. Salamanca, D. O. Salinas, and A. R. Messina, "Analysis of sub-synchronous torsional interactions with static VAR compensators-effect of network and load characteristics," in Proceedings of the IEEE Porto Power Tech, vol. 4, pp. 151-157, Porto, Portugal, September 2001.

[3] IEEE Subsynchronous Resonance Working Group, "Countermeasures to subsynchronous resonance problems," IEEE Transactions on Power Apparatus and Systems, vol. 99, no. 5, pp. 1810-1818, 1980.

[4] S. H. Hosseini and O. Mirshekar, "Optimal control of SVC for subsynchronous resonance stability in typical power system," in Proceedings of the IEEE International Symposium on Industrial Electronics (ISIE '01), vol. 2, pp. 916-921, Pusan, Republic of Korea, June 2001.

[5] O. Wasynczuk, "Damping subsynchronous resonance using reactive power control," IEEE Transactions on Power Apparatus and Systems, vol. 100, no. 3, pp. 1096-1104, 1981.

[6] Y.-Y. Hsu and C.-J. Wu, "Design of PID static VAr controllers for the damping of subsynchronous oscillations," IEEE Transactions on Energy Conversion, vol. 3, no. 2, pp. 210-216, 1988.

[7] C. A. Riversa S., A. R. Messina, D. Olguí S., and D. Ruiz V., "Analysis of subsynchronous torsional interactions with SVCs," Electric Power Components and Systems, vol. 31, no. 5, pp. 467481, 2003.

[8] S. H. Hosseini and O. Mirshekar, "Optimal control of SVC for subsynchronous resonance stability in typical power system," in Proceedings of the IEEE International Symposium on Industrial Electronics (ISIE '01), vol. 2, pp. 916-921, June 2001. 
[9] K. R. Padiyar and N. Prabhu, "Design and performance evaluation of subsynchronous damping controller with STATCOM," IEEE Transactions on Power Delivery, vol. 21, no. 3, pp. 13981405, 2006.

[10] S. Gupta, A. Arora, V. K. Panchal, and S. Goel, "Extended biogeography based optimization for natural terrain feature classification from satellite remote sensing images," in Proceedings of the 4th International Conference on Contemporary Computing (IC3 '11), pp. 262-269, Noida, India, August 2011.

[11] R. Mukherjee and S. Chakraborty, "Selection of the optimal electrochemical machining process parameters using biogeography-based optimization algorithm," The International Journal of Advanced Manufacturing Technology, vol. 64, no. 5-8, pp. 781-791, 2013.

[12] M. Ovreiu and D. Simon, "Biogeography-based optimization of neuro-fuzzy system parameters for diagnosis of cardiac disease," in Proceedings of the 12th Annual Genetic and Evolutionary Computation Conference (GECCO '10), pp. 1235-1242, July 2010.

[13] A. Hadidi and A. Nazari, "Design and economic optimization of shell-and-tube heat exchangers using biogeography-based (BBO) algorithm," Applied Thermal Engineering, vol. 51, no. 12, pp. 1263-1272, 2013.

[14] D. Simon, "Biogeography-based optimization," IEEE Transactions on Evolutionary Computation, vol. 12, no. 6, pp. 702-713, 2008.

[15] I. Boussaid, A. Chatterjee, P. Siarry, and M. Ahmed-Nacer, "Hybridizing biogeography-based optimization withdifferential evolution for optimal power allocation in wireless sensor networks," IEEE Transactions on Vehicular Technology, vol. 60, no. 5, pp. 2347-2353, 2011.

[16] P. K. Roy, S. P. Ghoshal, and S. S. Thakur, "Optimal reactive power dispatch considering flexible AC transmission system devices using biogeography-based optimization," Electric Power Components and Systems, vol. 39, no. 8, pp. 733-750, 2011.

[17] G. Lv, H. Cheng, W. He, J. Zhang, and G. Pan, "A low-EMI PWM approach for DC/AC inverter based on logistic chaotic map," International Journal of Power \& Energy Systems, vol. 27, no. 1, pp. 92-97, 2007.

[18] A. Müller, "On the performance of linear adaptive filters driven by the ergodic chaotic logistic map," International Journal of Bifurcation and Chaos in Applied Sciences and Engineering, vol. 22, no. 12, Article ID 1250290, p. 11, 2012.

[19] X. Xie, T. Yang, Q. Jiang, J. Lin, and Y. Wu, "Mechanism study on the mitigation of SSR with SVC," Automation of Electric Power Systems, vol. 32, no. 24, pp. 1-5, 2008. 


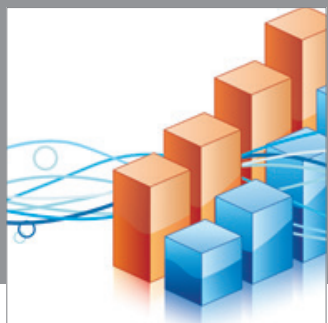

Advances in

Operations Research

mansans

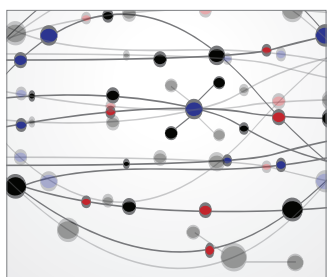

The Scientific World Journal
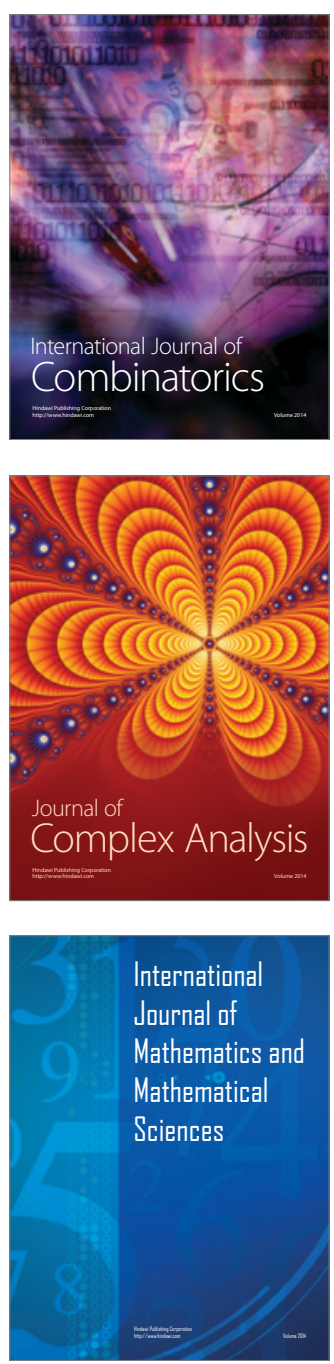
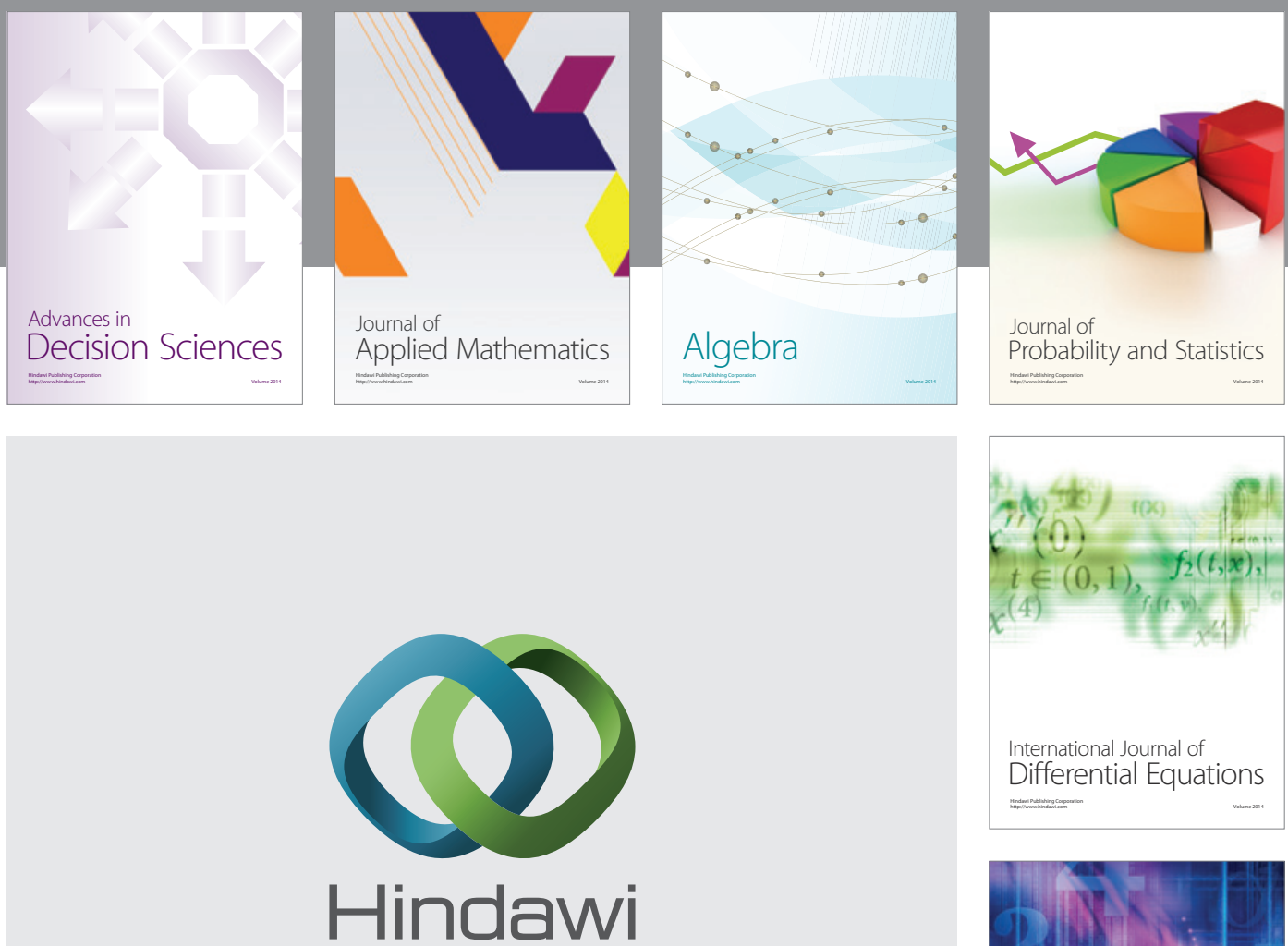

Submit your manuscripts at http://www.hindawi.com
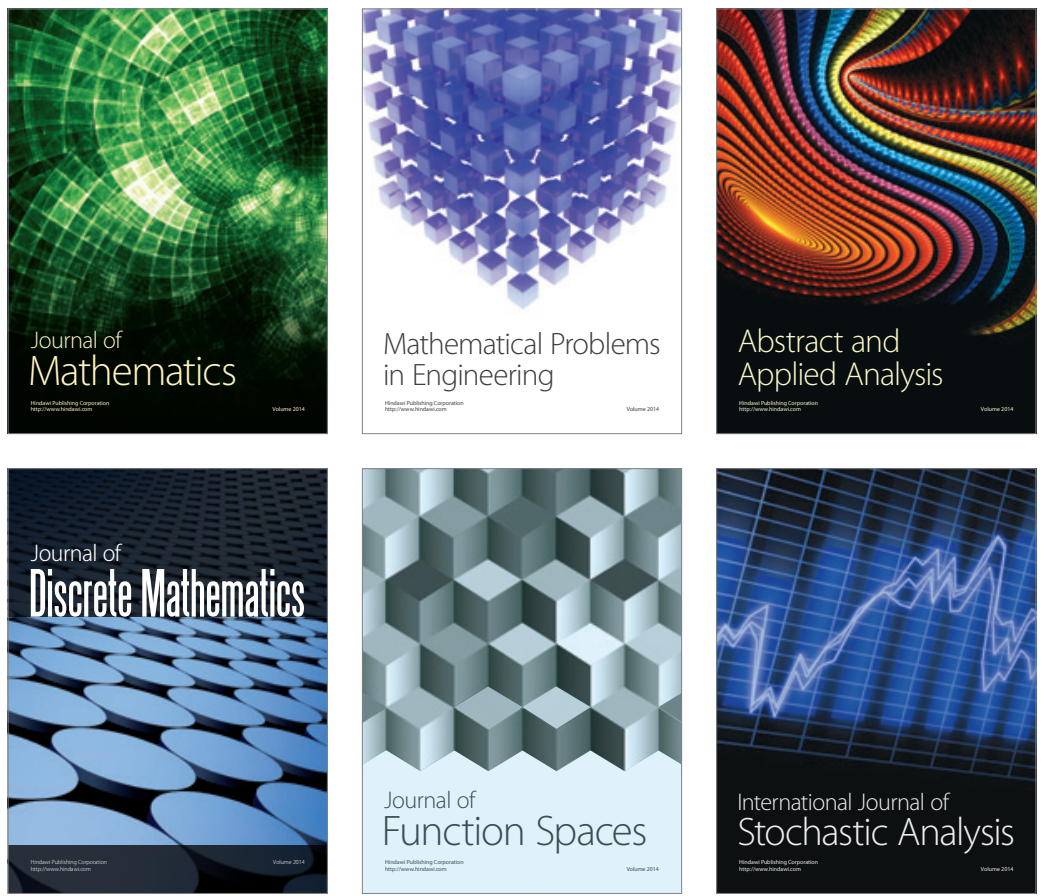

Journal of

Function Spaces

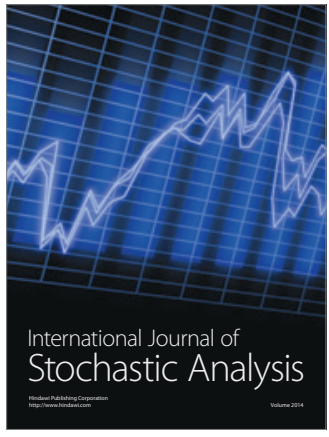

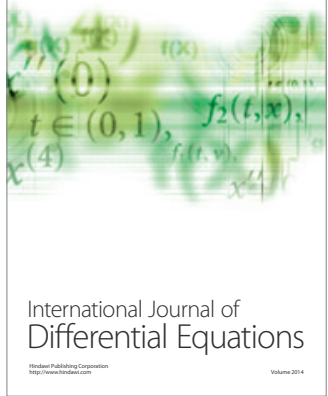
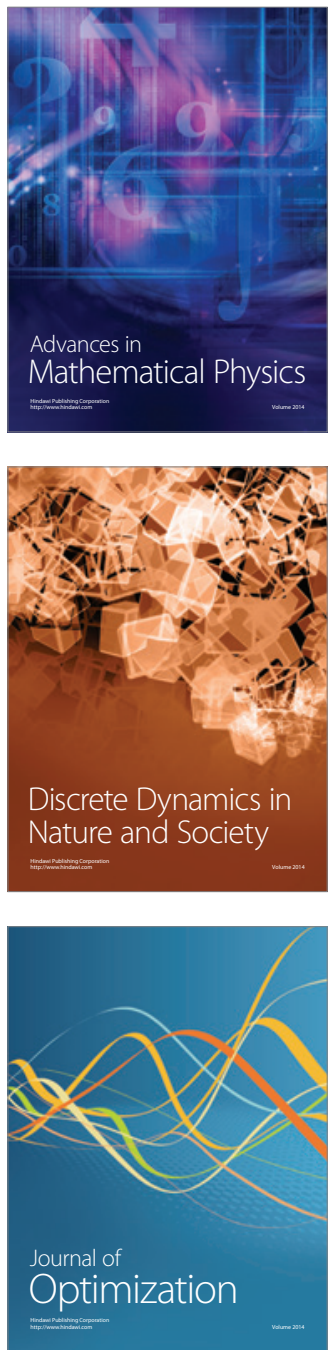